\title{
A High Speed Railway Wireless Broadband Access Network Based on Millimeter-wave 5G
}

\author{
Guoqing $\mathrm{Hu}^{1}, 2, \mathrm{a},{ }^{*}$, Jingjing Liu${ }^{1}$, Jun Pan ${ }^{1}$, Jin $\mathrm{He}^{1,2, b}$, Chunlai $\mathrm{Li}^{1}$, Huali You${ }^{1}$, \\ Zhijiong Wang ${ }^{1}$ and $Y$ ing $\mathrm{Yu}^{1}$ \\ ${ }^{1}$ Peking University Shenzhen SoC Key Laboratory, Peking University Shenzhen Institution, \\ Shenzhen, China \\ 2 School of Electronics Engineering and Computer Science, Peking University, Beijing, China \\ ahuking@pku.edu.cn, bfrankhe@pku.edu.cn \\ ${ }^{*}$ Corresponding author: huking@pku.edu.cn
}

Keywords: Millimeter-wave 5G, Mobile Communications, High Speed Railway Wireless Broadband Access Network.

\begin{abstract}
By the end of December 2017, China's high-speed railway (HSR) has a total mileage of 25 thousand $\mathrm{km}$. However, providing reliable and stable wireless broadband access service for HSR passengers has been a major challenge in the communications industry. This paper proposes a HSR wireless broadband access network based on millimeter-wave 5G. The millimeter-wave 5G can support the mobility of $500 \mathrm{Km} / \mathrm{h}$, tens of Gbps peak rate and the end to end delay can be reduced to several millisecond. It can fully meet the requirements of low delay, high reliability and high bandwidth in the HSR scene.

\section{一种基于毫米波 $5 \mathrm{G}$ 的高铁无线宽带接入网

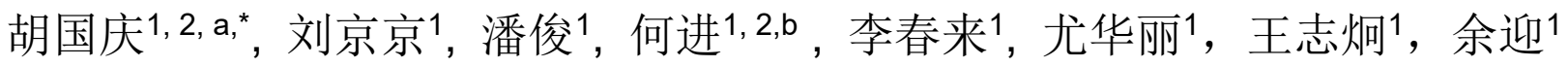 \\ 1 北京大学深圳研究院系统芯片设计重点实验室, 深圳, 中国 \\ 2 北京大学信息科学技术学院, 北京, 中国 \\ ahuking@pku.edu.cn, bfrankhe@pku.edu.cn \\ *通讯作者: huking@pku.edu.cn}

关键词: 毫米波5G；移动通信；高铁无线宽带接入网。

中文摘要. 截止至2017年12月, 中国高速铁路HSR (High Speed Railway) 总营业里程已达到 2.5 万公里。然而, 为时速达到 $350 \mathrm{~km} / \mathrm{h}$ 的高铁乘客提供可靠而稳定的无线宽带接入一直是全球 通信业的重大挑战, 为此本文提出了一种基于毫米波 $5 \mathrm{G}$ 的高铁无线宽带接入网方案。毫米波 $5 \mathrm{G}$ 接入方案可以支持 $500 \mathrm{Km} / \mathrm{h}$ 的移动性, 峰值速率达到几十 $\mathrm{Gbps}$, 端到端的时延降低到毫秒 量级，完全可以满足高铁场景下低时延、高可靠、高带宽的要求。

\section{1. 引言}

近年来，高速铁路在全世界范围内得到了大规模的普及。截止至2017年12月，中国高速 铁路HSR (High Speed Railway) 总营业里程 2.5 万公里, 占世界高铁运营里程 $60 \%$ 以上。随着 中国经济的发展和智能手机终端的大规模普及, 越来越多的高铁乘客希望能够在高铁列车上 享受到无线宽带接入服务。然而, 为时速达到 $350 \mathrm{~km} / \mathrm{h}$ 的高速移动台提供可靠而稳定的无线 宽带接入一直是全球通信业的重大挑战。目前, 国内外针对高铁无线宽带接入网的网络架构 
所采用的核心技术主要有以下几种: 卫星链路、泄漏同轴电缆链路、无线局域网WLAN (Wireless Local Area Networks) 链路、WiMAX (Worldwide Interoperability for Microwave

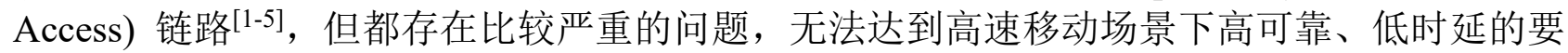
求。

随着移动互联网和物联网业务的爆发式增长, 第五代移动通信系统5G应运而生。5G不仅 将满足人们居住、休闲、娱乐等各种领域的业务需求, 更能为高铁等超高流量密度、超高连 接数密度、超高移动性特征的场景提供高清视频、在线游戏等优质业务体验。如图1所示, 为 $5 \mathrm{G}$ 的主要性能指标, 从图中可以看出, $5 \mathrm{G}$ 需要支持 $500 \mathrm{Km} / \mathrm{h}$ 的移动性, 峰值速率达到几十 Gbps, 端到端的时延降低到毫秒量级, 完全可以满足高铁无线宽带接入网的用户需求。因此, 本文提出了一种基于毫米波 $5 \mathrm{G}$ 的高铁无线宽带接入网, 可为高铁旅客提供高质量的无线宽带 接入服务。

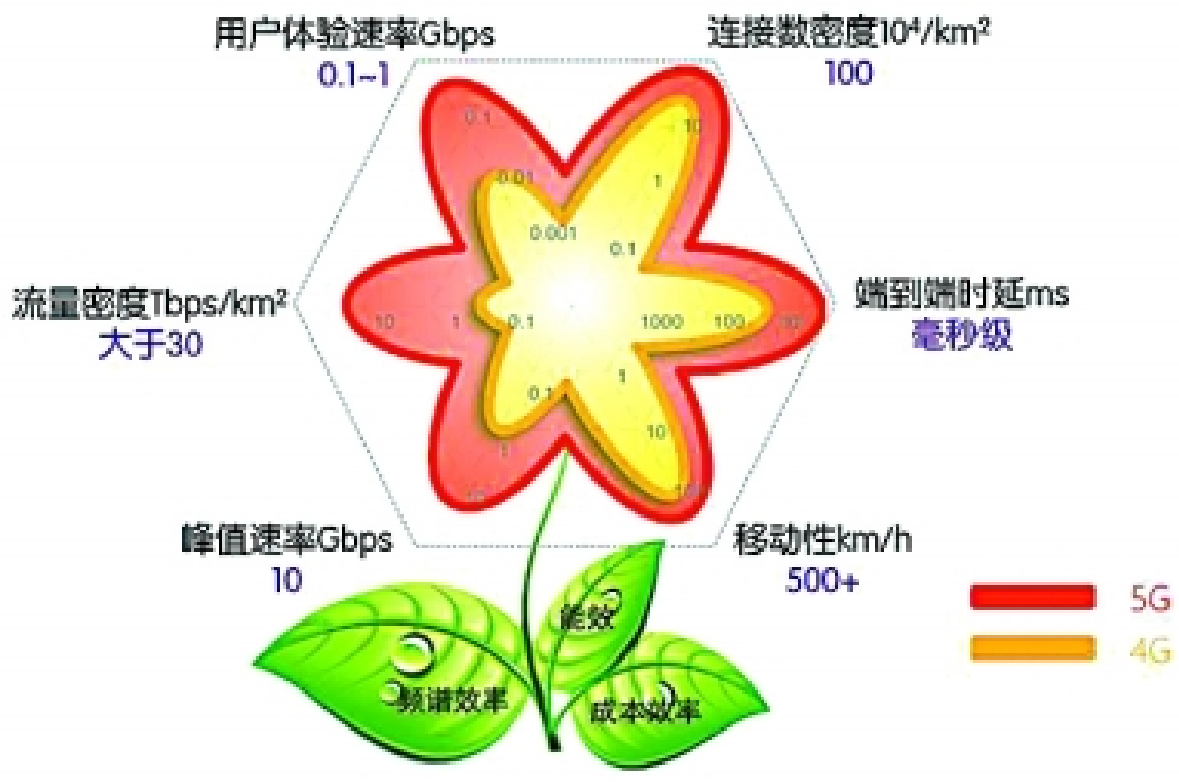

图 $15 \mathrm{G}$ 关键性能指标 ${ }^{[6]}$

\section{2. 基于毫米波 $5 G$ 的高铁无线宽带接入网架构}

如图2所示, 基于毫米波 $5 \mathrm{G}$ 的高铁宽带接入网ETAN (Express Train Access Network) 由三 部分组成：负责地面到列车之间连接的毫米波RoF（Radio over Fiber）架构，负责车厢内部无 线连接的局域网LAN(Local Access Network)，捕捉列车移动状态和位置信息的光传感网。接 下来，我们分别介绍三部分的结构及其功能。

毫米波RoF架构及其功能：外部移动通信网、有线电视网、因特网和列车控制信号网通 过一个业务汇聚网关接入到RoF系统的中心站 $\mathrm{CS}$ 。这些业务信息通过RoF系统的光纤链路传 输到远端天线单元RAU( Remote Antenna Unit)。在RAU内, 经过光电转换恢复成毫米波 $5 \mathrm{G}$ 无 线信号, 放大后通过天线发送到列车顶部的接入点AP。之后再通过AP连接的列车内部局域网 LAN传送到用户设备端UE (User Equipment)。中心站内的光切换单元负责完成不同蜂窝小区 间的切换, 光传感信息处理单元LS-DPS (Light Sensor Data Processing System) 负责汇聚光传 感网络的数据, 分析出列车的移动状态和位置信息, 为切换判决提供决策信息。

列车内部局域网LAN：接收到远端天线单元RAU发送来的毫米波5G业务信息后，首先经 过数据处理, 将各种业务信息转换为车厢内用户端UE可以直接接收的信号。之后, 通过有线 或者无线的方式在车厢内为用户提供电视业务、移动通信业务、因特网业务, 同时将列车控 制信息传送给列车驾驶员和其他列车控制人员。 
光传感网络：光传感器部署在相邻小区间的重叠区域（即切换区域）。这些传感器用来 捕捉列车的移动速度和位置等信息, 这些信息会汇聚到中心站CS中的光传感信息处理单元 LS-DPS, 为切换判决提供决策信息。

\section{LS-DPS: Light Sensor Data Processing System}

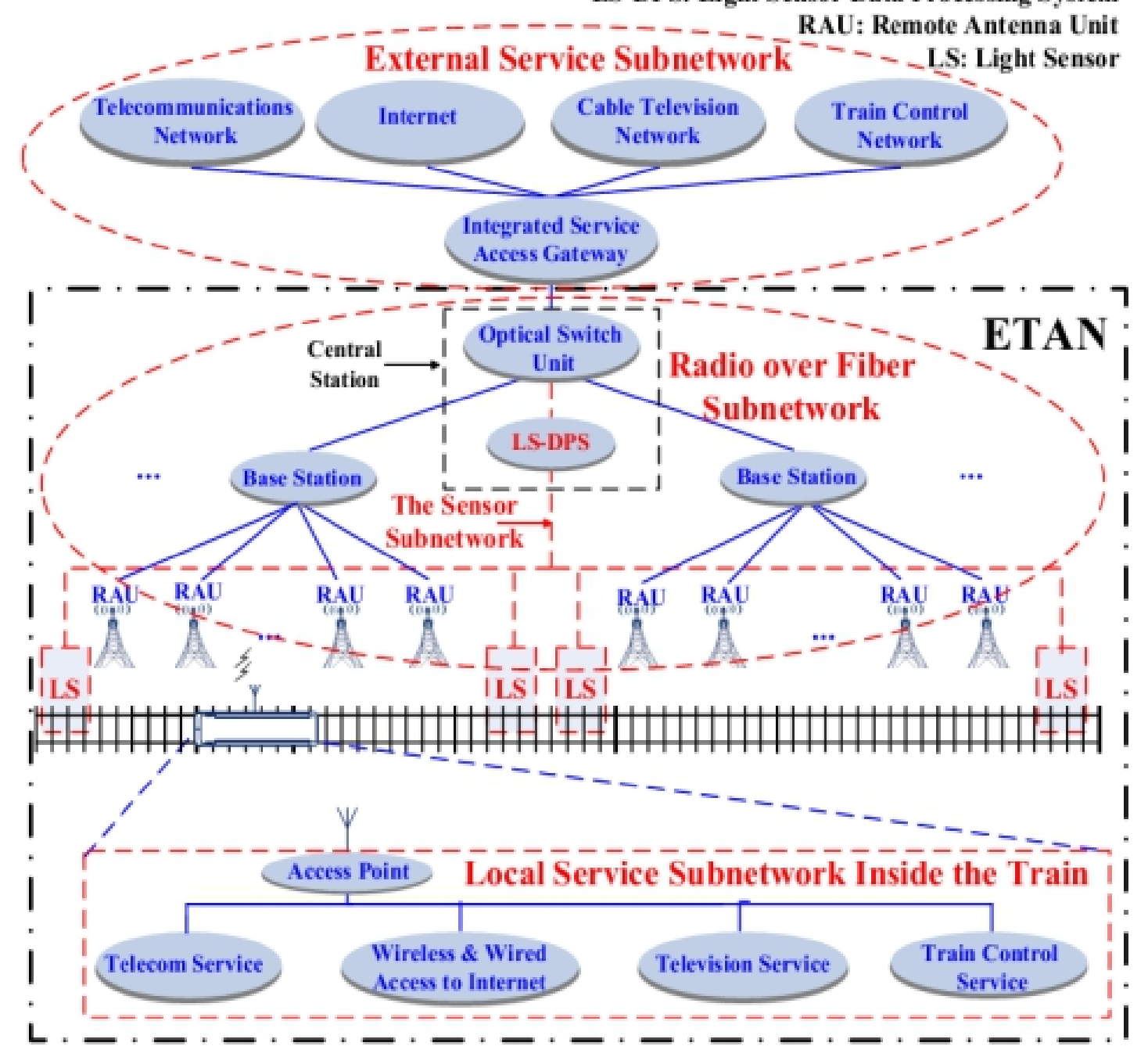

图2 基于毫米波 $5 \mathrm{G}$ 的高铁宽带接入网架构

高铁宽带无线接入网采用毫米波 $5 \mathrm{G} \mathrm{RoF}$ 架构，具有如下优势:

第一，毫米波RoF技术可以有效降低系统成本。采用RoF技术，可以将数量庞大的交换、 控制以及信号再生设备集中到中心站 $\mathrm{CS}$, 基站BS只需完成光电转换。通过这种方式将价格昂 贵的设备集中在中心站, 而让多个远端天线单元共享这些设备, 进而大幅降低了基站的能源 消耗和建设成本。

第二, 毫米波RoF系统内, 在同一基站下的远端天线单元RAU之间无需进行切换, 这样 可以大幅降低高铁宽带接入网中的切换次数。同一基站下的RAU使用的是相同的频率, 将小 区覆盖拉成了紧挨高铁沿线的线性覆盖, 不同的RAU之间相当于起到了一个中继器的作用, 所以在同一基站下的RAU之间无需进行切换, 这样就既能实现蜂窝小区的小半径覆盖, 又可 以大幅降低了切换次数。有效解决了小覆盖半径与切换问题之间的矛盾。

第三，毫米波RoF系统可以提供高达几十Gbps的传输速率，可以满足高铁场景下高用户 密度、大带宽的需求。在高速列车上存在数以千计的用户, 以16节 $\mathrm{CRH}$ 系列为例, 单次列车 载客量在 1200 人以上。以每位用户提供 $5 \mathrm{Mbps}$ 的传输速率来计算, 整个列车的总传输速率也 需要在 $6000 \mathrm{Mbps}$ 以上。如此高的传输速率和带宽需求, 对于其他高铁接入技术而言是一种极 其严峻的挑战, 而对于毫米波 $5 \mathrm{G} \mathrm{RoF}$ 系统却可以满足我们的需求, 目前毫米波 $5 \mathrm{G}$ 通信系统传 输速率已突破70 $\mathrm{Gbps}^{[7]}$ 。 


\section{3. 结束语}

为时速达到 $350 \mathrm{~km} / \mathrm{h}$ 的高速移动台提供可靠而稳定的无线宽带接入一直是全球通信业的 重大挑战, 为此本文提出了一种基于毫米波 $5 \mathrm{G}$ 的高铁无线宽带接入网方案。毫米波 $5 \mathrm{G}$ 接入方 案可以支持 $500 \mathrm{Km} / \mathrm{h}$ 的移动性, 峰值速率达到几十 $\mathrm{Gbps}$, 端到端的时延降低到毫秒量级, 完 全可以满足高铁场景下低时延、高可靠、高带宽的要求, 可为高铁乘客提供高清视频、在线 游戏、宽带接入等优质业务体验。

\section{致谢}

本文由深圳市个人创客项目（GRCK2017042415235934），深圳市基础研究项目 （ JCYJ20170817113758285 、 JCYJ20170307164201104、 JCYJ20160329161334453 、 JCYJ20170817112527562 、JCYJ20170412153845293 、 JCYJ20170307164247428 、 JCYJ20170817113844300 、 JCYJ20170817112708243 、 JCYJ20170817113029596 、 JCYJ20170817112848591、JCYJ20170412153812353、JCYJ20170307172513653），中国博 士后基金面上资助项目（2017M620523、2017M622619），深圳市技术攻关项目 （JSGG20170414140411874），国家自然科学基金项目（61574005）, 深港产学研基地产学 研项目（场效应太赫兹器件的关键技术研究）资助。

\section{References}

[1] Guoqing $\mathrm{Hu}$, Anpeng Huang, Tammy Chang, Xiang Cheng, Hequan Wu, LinzhenXie, Anshi $\mathrm{Xu}$, Zhangyuan Chen, "A Sensor-Based Seamless Handover Solution for Express Train Access Networks (ETANs),” IEEE Communications Letters, vol.16, no.4, pp. 470-472, April 2012.

[2] Guoqing $\mathrm{Hu}$, Anpeng Huang, Ruisi He, Bo Ai, Zhangyuan Chen, "Theory analysis of the handover challenge in Express Train Access Networks (ETAN)", China Communications, vol. 11, no.7, pp. 92-98, July 2014.

[3] Cheng-Xiang Wang, Ammar Ghazal, Bo Ai, et al., "Channel Measurements and Models for High-Speed Train Communication Systems: A Survey," IEEE Communications Surveys \& Tutorials, vol. 18, no. 2, pp. 974-987, 2016.

[4] Guoqing $\mathrm{Hu}$, Guangjin Ma, Chuanlai Li, Yongchi Xu, Jin He, Ying Yu, Yandong He, “An Empirical Analysis of the Radio Propagation Characteristics in High-Speed Railway Environment", 2017 International Conference on Electrical, Mechanical and Computer Engineering (ICEMCE 2017), IET, Chengdu, China, pp.1-4, May 25-28, 2017.

[5] Guoqing Hu, Jin He, Guangjin Ma, Chunlai Li , Ying Yu, "Summary of the Study on High Speed Railway Access Network", The 2017 2nd International Conference on Electrical Technology and Mechanical Engineering (ICETME 2017), Guangzhou, China, pp.1-4, July 15-16, 2017.

[6] China Academy of Information and Communications Technology, "The general development trend of 5G", 2015.12.

[7] J. Huang, C.-X. Wang*, R. Feng, J. Sun, W. Zhang, and Y. Yang, "Multi-frequency MmWave massive MIMO channel measurements and characterization for $5 \mathrm{G}$ wireless communication systems,” IEEE J. Sel. Areas Commun., vol. 35, no. 7, pp. 1591-1605, July 2017. 